DrAft VERSION NOVEMBER 3, 2018

Preprint typeset using $\mathrm{L}^{A} \mathrm{~T}_{\mathrm{E}} \mathrm{X}$ style emulateapj v. 11/10/09

\title{
A NEW DELIVERY ROUTE TO GALACTIC NUCLEI: WARM HALO CLOUD IMPACTS
}

\author{
BARry MCKERnan ${ }^{1,2}$, ArIYeH MALleR ${ }^{3}$, K.E.SAAVIK Ford ${ }^{1,2}$ \\ Draft version November 3, 2018
}

\begin{abstract}
We propose a new mechanism for the delivery of gas to the heart of galactic nuclei. We show that warm halo clouds must periodically impact galactic centers and potentially deliver a large $(\sim$ $\left.10^{4-6} M_{\odot}\right)$ mass of gas to the galactic nucleus in a singular event. The impact of an accreting warm halo cloud originating far in the galactic halo can, depending on mixing, produce a nuclear starburst of low metallicity stars as well as low luminosity accretion onto the central black hole. Based on multiphase cooling around a $\Lambda \mathrm{CDM}$ distribution of halos we calculate the nuclear impact rate, the mass captured by the central black hole and the fraction of active nuclei for impacting cloud masses in the range $10^{4}-10^{6} M_{\odot}$. If there is moderate braking during cloud infall, our model predicts an average fraction of low luminosity active nuclei consistent with observations.
\end{abstract}

\section{INTRODUCTION}

Activity in galactic nuclei is fuelled by a reservoir of low angular momentum gas, but it is unclear how such reservoirs build up. Fast outflows from OB stars, supernovae or AGN activity can clear out nuclear ISM (Ciotti \& Ostriker 1997; Schawinski et al. 2007; McKernan et al. 2007; Schartmann et al. 2009). Gas in the nucleus can also be quickly consumed by star formation (Nayakshin, Cuadra \& Springel 2007) or driven outwards by positive gravitational torques (Garcia-Burillo et al. 2005). If the gas in the reservoir originates outside the nucleus, multiple mechanisms operating on different distance- and time-scales, such as bars feeding nuclear rings (Krolik 2001), radiation drag (Kawakatu et al. 2003) or interactions (Vittorini et al. 2005) are required. From observations, around $1 / 3$ of all galactic nuclei in the local Universe exhibit low luminosity nuclear activity ( $\mathrm{Ho} 2008)$, so the mechanism promoting nuclear gas build up is likely to be simple and on-going.

In this letter we propose a new mechanism for delivery of gas to the galactic nucleus. We show that the impact of a warm halo cloud (WHC), containing $\sim 10^{4-6} M_{\odot}$, on the central regions of a galaxy will fuel nuclear activity. We develop a simplistic model of this phenomenon to demonstrate its likely importance. While there remains considerable uncertainty in WHC parameters, we show that for plausible input parameters, a direct hit by a single WHC on the center of a galaxy will supply fuel for star formation and radiatively inefficient accretion onto the central black hole. Since WHC bombardment of galaxies must occur, some fraction of the low luminosity activity observed in galactic nuclei must be due to WHC impacts on galactic nuclei.

\section{WARM HALO CLOUDS}

\footnotetext{
bmckernan@amnh.org

${ }^{1}$ Department of Science, Borough of Manhattan Community Collge, City University of New York, New York, NY 10007

2 Department of Astrophysics, American Museum of Natural History, New York, NY 10024

${ }^{3}$ Department of Physics, New York City College of Technology, City University of New York, New York, NY 11201
}

The assembly of gaseous halos around galaxies naturally produces a multiphase medium with warm halo clouds (WHCs) embedded in a low density hot gas halo (Maller \& Bullock 2004). WHCs could be the local analogue of the high redshift Lyman limit systems (Blitz et al. 1999). In the halo of our own Galaxy, numerous warm clouds are observed with velocities that are inconsistent with Galactic rotation (e.g. Peek et al. 2007). Around several other galaxies, HI clouds of $10^{5-8} M_{\odot}$ have been detected (e.g. Chynoweth et al. 2008; Boomsma et al. 2008). Extended HI structures (up to $\sim 10^{10} M_{\odot}$ ) are observed out to $\sim 100 \mathrm{kpc}$ around early-type galaxies (Oosterloo et al. 2007). So, a population of WHCs containing $\sim 10^{4-6} M_{\odot}$ per cloud, may be common around most galaxies (see e.g. Maller \& Bullock 2004). Around our Galaxy, two basic models can account for the properties of observed high velocity clouds (HVCs): an accretion model based on WHCs (e.g. Blitz et al. 1999; Maller \& Bullock 2004) or a Galactic fountain model (Shapiro \& Field 1976). Accreting WHCs should dominate the mass of clouds in the Galactic halo so in this work we shall concentrate on the effects of an accreting WHC impact on galactic nuclei. Much of the following discussion also applies to galactic fountain clouds, although that population will have higher metallicity, less mass, smaller radius and lower velocity on average, and will be less numerous.

\section{WHC IMPACT WITH GALACTIC CENTER}

Large uncertainties exist concerning cloud trajectories around our own Galaxy (e.g. Thom et al. 2008). While we might naively expect radial trajectories for WHCs, cloud trajectories may become randomized close to a galaxy. Clouds close to the disk become tidally disrupted (Blitz et al. 1999; Maller \& Bullock 2004), or deflected by magnetic fields (Kwak, Shelton \& Raley 2009), or disrupted by the Kelvin-Helmholz instability (KHI) (Heitsch \& Putman 2009) and the fragments dispersed about the disk, whereupon the previous cloud trajectory may be irrelevant. Since we have no way of predicting actual cloud trajectories, in the discussion below we simply assume that clouds impact the disk randomly.

We start by assuming a initial population of $N_{c l}$ clouds on random trajectories raining down on a galaxy of ra- 
dius $R_{\text {gal }}$. As long as the WHC radius is larger than the nuclear region under consideration, the rate of impact $\left(d N_{c l} / d t\right)$ of infalling $\mathrm{WHC}$ of radii $r_{c l}$ on the galactic center is

$$
d N_{c l} / d t=0.25\left(r_{c l} / R_{g a l}\right)^{2} / \tau
$$

where $\tau$ is the typical cloud infall time and the cloud material is assumed to arrive within $0.5 r_{c l}$ of the galactic center. We calculate the cloud impact rate for multiphase cooling around a $\Lambda \mathrm{CDM}$ distribution of halos based on the model of Maller \& Bullock (2004). In this model the total mass in WHCs is based on the mean free path of the clouds and the cloud properties we use are the average of some distribution in the halo. First we calculate the average nuclear impact rate, using $R_{\text {gal }}=0.15 R_{\text {cool }}$, based on conservation of angular momentum (Fall \& Efstathiou 1980), where $R_{\text {cool }}$ is the cooling radius (White \& Frenk 1991). We simplify our calculation by assuming that the WHC does not fragment and that infall time is $\tau=R_{\text {cool }} / V_{\max }$ where $V_{\max }$ is the maximum circular velocity in the halo. The results are shown in Fig. 1(a), where we plot impacts/Gyr versus $V_{\max }$ for impacting cloud masses of $10^{4}, 10^{5}, 10^{6} M_{\odot}$. Evidently the impact rate is relatively flat with $V_{\max }$ (or equivalently black hole mass). Note that a nuclear impact rate of $\sim 5 /$ Gyr from Fig. 11(a) corresponds to a galactic impact rate of $\sim 200 /$ Gyr or a mass inflow rate of $\sim 1 M_{\odot} /$ yr if the typical impactor mass is $\sim 10^{6} M_{\odot}$. This is approximately the low metallicity inflow rate required to explain the so-called 'G-dwarf problem' (e.g. Edmunds 1990, and references therein) .

\section{DELIVERING MATERIAL TO THE GALACTIC NUCLEUS}

Although observational constraints of cloud impacts are not strong (Lockman et al. 2008), we can consider the consequences of WHC impact on a galactic nucleus, guided by simulations of HVC impacts with the disk (see e.g. Kwak, Shelton \& Raley 2009; Heitsch \& Putman 2009, and references therein). The same basic sources of cloud fragmentation in the halo and disk will apply to a WHC falling into a galactic bulge. If galactic nuclei have relatively strong magnetic field strengths in general (e.g. Metzger et al. 1996), the magnetic fields will act as a strong brake on infalling WHCs and the cloud may fragment.

Instabilities in the infalling cloud have growth times of the order of the timescale on which shocks cross the cloud. This cloud crushing timescale, $t_{c c}$, is given by (Klein, McKee \& Colella 1994)

$$
t_{c c}=\left(\frac{n_{c l}}{n_{m}}\right)^{\frac{1}{2}} \frac{r_{c l}}{v_{c l}}
$$

where $n_{c l}$ and $n_{m}$ are the densities of the WHC and the surrounding medium respectively and $v_{c l}$ is the relative velocity of the WHC and the surrounding medium. If the WHC is accreting from a very large distance, $t_{c c}$ can be less than the infall time ( $\sim$ Gyrs) and the cloud can fragment in the halo. Indeed many of the HVCs presently observed may be fragments of initially much larger accreting WHCs. Galactic haloes are hot and diffuse (e.g. Strickland et al. 2004; McKernan et al. 2004, and references therein) and the disruption of a WHC in the halo could create an infalling stringy association of clouds like the HVC A and $\mathrm{C}$ complexes. If the trajectory of such a stringy cloud complex intercepted the galactic nucleus then most of the WHC mass could be delivered to the region around the central supermassive black hole.

Sufficiently dense and fast clouds falling through the halo and then the bulge ISM will first experience deceleration and compression (pancaking), followed by an expansion of the shocked cloud downstream, then lateral expansion and finally cloud destruction when instabilities and differential forces fragment the cloud. This entire process takes place over a few $t_{c c}$ (see e.g. Klein. McKee \& Colella 1994; Kwak. Shelton \& Ralev 2009). Compression will increase the WHC density as the cloud pancakes (Kwak. Shelton \& Raley 2009; Heitsch \& Putman 2009). Small, fast, dense fragments of cloud can also sweep up large amounts of gas and be shocked (Wakker \& van Woerden 1997). The transit time for a WHC through a bulge of radius $R_{b}$ to the galactic center is $\sim R_{b} / \eta v_{c l}$ where $\eta=[0,1]$ is a coefficient incorporating slowdown due to drag and magnetic fields. For a galaxy the size of our $\operatorname{own}\left(R_{b} \sim 3 \mathrm{kpc}\right)$, a cloud with $v_{c l} \sim 100 \mathrm{~km} \mathrm{~s}^{-1}$ will take $\sim 30 / \eta \mathrm{Myr}$ to cross the bulge and reach the center.

But will the cloud survive this long? Outside of the inner $\sim 0.5 \mathrm{kpc}$, the bulge ISM in our own galaxy has very low density $\left(<10^{-3} \mathrm{~cm}^{-3}\right)$ Tang et al. (2009). At these densities, the cloud crushing timescale is $>100 \mathrm{Myrs}$, far larger than the transit time through this part of the bulge. Within $0.5 \mathrm{kpc}$ of the Galactic center the bulge ISM density increases to $\sim 0.01 \mathrm{~cm}^{-3}$ (Tang et al. 2009). Here the crushing timescale is $\sim 30 / \eta \mathrm{Myr}$, short enough that the WHC will fragment in this region. So, WHCs could survive until they reach the central regions, whereupon fragmentation is likely.

\section{GRAVITATIONAL CAPTURE BY THE CENTRAL BLACK HOLE}

Evidently a WHC cloud impact could deliver a large quantity of moderate density gas to a galactic nucleus in a single cloud infall. But what happens once fragments of a cloud of mass $M_{c l} \sim 10^{4-6} M_{\odot}$ arrives in the central few tens of pc of a galaxy? Low angular momentum fragments of the WHC will be captured by the central black hole out to a radius $\left(R_{e s c}\right)$ where their velocity is below the escape speed. Thus the mass captured is

$$
M_{c a p t}=M_{c l} \frac{R_{e s c}^{2}}{r_{c l}^{2}}=M_{c l} \frac{\left(2 G M_{B H}\right)^{2}}{\left(\eta v_{c l}\right)^{4} r_{c l}^{2}} .
$$

We calculate the average $M_{\text {capt }}$ for clouds of mass $10^{4-6} M_{\odot}$ impacting galactic nuclei. We estimate black hole mass using the $M_{B H}-\sigma$ relation from Gultekin et al. (2009) and we assume that the bulge velocity dispersion and maximum circular velocity are related as in Ferrarese (2002) so that

$$
\log \left(\mathrm{M}_{\mathrm{BH}}\right)=5.05 \log \left(\mathrm{V}_{\max }\right)-4.41
$$

which should be valid over the range $\sigma_{b} \sim 70-350 \mathrm{~km}$ $\mathrm{s}^{-1}$. We allowed different values of braking $(\eta)$, ranging from none $(\eta=1)$ to strong $(\eta=0.1)$ which are shown in Fig. 1(b). Clearly the braking parameter determines when the average mass captured reaches the total cloud mass. Our model predicts that the Edding- 
ton ratio will be greatest near a critical value of $V_{\max }$, where the average mass captured reaches the total cloud mass. Evidently a nuclear strike on a black hole with mass $<10^{7} M_{\odot}$ yields much less mass available for accretion onto the black hole, even for large braking $(\eta=0.1)$. However, a much larger fraction of $M_{c l}$ could be captured if the infalling cloud fragments into a stringy cloud complex in the galactic halo, effectively reducing $r_{c l}$ for a given $V_{\max }$. Cloud material not captured by the black hole will instead be gravitationally captured by the bulge. In this case, the material will either persist as part of the bulge ISM or will go down the route of star formation (with some unknown level of mixing). This is consistent with observations of nuclear activity due to $H_{\mathrm{II}}$ regions predominating in galaxies with smaller bulges and therefore smaller mass central black holes ( $\mathrm{Ho} 2008$ ).

\section{ACTIVITY IN GALACTIC NUCLEI}

What sort of nuclear activity will result from a WHC impact? Once moderately dense, low angular momentum fragments of the WHC arrive in the galactic nucleus, star formation is likely, although dependent on poorly constrained variables such as cooling rate and mixing with nuclear gas. The arrival of a large quantity of infalling gas may also initiate activity via shocks or gradual acceleration of GMCs (Hopkins \& Hernquist 2006). In our model, shocked nuclear GMCs or the highest density, shocked fragments resulting from a WHC impact could generate a nuclear OB association within $\sim 10 \mathrm{Myr}$ after impact (Clark et al. 2005). A direct hit by a WHC fragment on the central black hole may result in a phase of low luminosity Bondi accretion.

Kauffmann \& Heckman (2009) find two distributions of Eddington ratios among active galactic nuclei in the local Universe. Their first, a lognormal distribution, centered around a few percent of Eddington, is associated with nuclear star formation, dominates among black holes $<10^{8} M_{\odot}$ and depends critically on feedback from luminous accretion. The second distribution is a powerlaw which occurs in galaxy bulges where there is little or no ongoing star formation. We will only model the second mode since the first mode has a complex association with star formation, stellar evolution and AGN feedback. Although the distribution of $\mathrm{dM} / \mathrm{dt}$ in this second accretion mode is a powerlaw, we will use the average value from our $\Lambda \mathrm{CDM}$ calculations to get an average activity fraction. We calculate the average fraction of galaxies of a given $V_{\max }$ that are active for impactors of mass $10^{4-6} M_{\odot}$ and a range of braking and cloud densities. The lifetime of Bondi accretion is $\tau_{B}=M_{\text {capt }} /(d M / d t)$ where

$$
d M / d t \approx 10^{-3} n M_{8}^{2}\left(\eta v_{200}\right)^{-3} M_{\odot} / y r .
$$

Here $n$ is the average density of the accreting gas, $M_{8}$ is the black hole mass in units of $10^{8} M_{\odot}$ and $v_{200}$ is an average combination of gas speed and gas sound speed in units of $200 \mathrm{~km} / \mathrm{s}$ (Ho 2008). The average gas density around the black hole is fairly unconstrained in our model and clearly there is trade off between more massive clouds that take longer to consume and higher gas densities which are consumed more quickly. Nevertheless, in this model the lowest average $\mathrm{dM} / \mathrm{dt}$ is $\sim 10^{-5} M_{\odot} / \mathrm{yr}$ around a $10^{8} M_{\odot}$ black hole, assuming $\eta, v_{200} \sim 1$ and $n \sim 0.01 \mathrm{~cm}^{-3}$. In Fig. 1(c) we show some average values of braking and gas density that yield reasonable models where on average $1 / 3$ of galactic nuclei are active. For $10^{4} M_{\odot}$ clouds this requires $n=0.004 \mathrm{~cm}^{-3}$, while for $10^{6} M_{\odot}$ clouds with some braking this requires $n=0.03 \mathrm{~cm}^{-3}$, and with no braking, $n=0.01 \mathrm{~cm}^{-3}$. Recall we are modelling the powerlaw accretion mode of Kauffmann \& Heckman (2009), which is significant among black holes of mass $>10^{8} M_{\odot}$. Therefore, from Fig. 1(b),(c), our model requires $\eta>0.5,0.3$ for impactor clouds of masses $10^{4} M_{\odot}$ and $10^{6} M_{\odot}$ respectively. Note that in all cases the plot shows a characteristic shape where the average activity first increases as the amount of the cloud captured increases and then decreases as the accretion rate increases for the same amount of captured cloud. Our model predicts that radiatively inefficient Bondi accretion should be more commonly detected around larger mass black holes, which is consistent with observations of LINER activity in the local Universe (e.g. Schawinski et al. 2007; Ho 2008; Kauffmann \& Heckman 2009). Future observations of low luminosity activity in galactic nuclei as a function of $V_{\max }$ will be able to rule out certain parameters. Of course if $\eta$ or $n$ depend on $V_{\max }$ then the shapes of the curves in Fig. 1(c) might differ. MHD simulations of cloud fragmentation in the nucleus are required to establish whether the distribution of densities and braking can in fact generate a powerlaw distribution of dM/dt (Kauffmann \& Heckman 2009) and we intend to do this in future work. Note that our model of Bondi accretion is unlikely to apply for $M_{\text {capt }}<M_{c l}$ since the dominant activity in that case would be nuclear star formation, which is consistent with observations of smaller mass central black holes (Ho 2008). Ciotti \& Ostriker (2007) argue that luminous accretion can occur in the aftermath of a nuclear starburst. This may yield the lognormal distribution of Eddington ratio observed in a fraction of galactic nuclei (Kauffmann \& Heckman 2009), but establishing this is far beyond the scope of the present work.

We expect the number of WHCs to increase with redshift as the gas accretion rate onto galaxies increases. We naively expect the number of WHC clouds and therefore the average cloud impact rate to scale with star formation rate or $\propto(1+z)^{3.1}$ from $z=0$ to $z=1$ (Doherty et al. 2006), corresponding to a rate of $\sim 1 / 30 \mathrm{Myr}$ for MilkyWay sized galaxies at $z \sim 1$. On these timescales, luminous nuclear activity could result if radiatively efficient accretion occurs onto the central supermassive black hole. A strongly decreasing Eddington ratio over cosmic time (e.g. Vittorini et al. 2005; Shankar et al. 2009; Shen 2009) could naturally produce a high duty cycle at $z \sim 0$, consistent with Fig. 1(c). At even earlier epochs $(z>2.5)$, the bulk of the growth of supermassive black holes occurs via mergers in massive protogalactic halos $\left(\sim 10^{11-13} M_{\odot}\right)$ (Cavaliere \& Vittorini 2002). At that time, gas accretion is primarily on random trajectories, so nuclear fueling by WHC impact would be at its maximum. A natural bridge can then be made to the time before hot halos are established (Kerĕs et al. 2005; Dekel et al. 2009), when gas accretion is all in the form of infalling gas. In the present epoch, our model predicts that clustering of activity should be random for similar sized galaxies (comparable $V_{\max }$ ), since cloud impacts are random. This is consistent with observations of clus- 


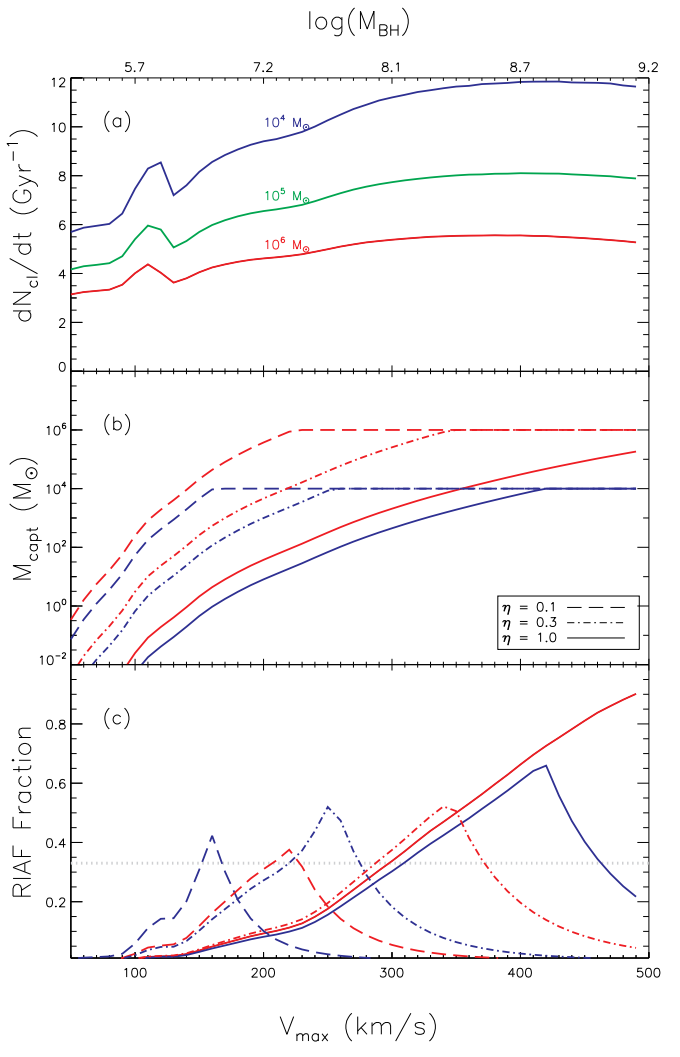

FIG. 1.- Plots of: (a) Average WHC impact rate (number/Gyr) on galactic nuclei versus maximum halo circular velocity $(\mathrm{km} / \mathrm{s})$ for impactor cloud masses of $10^{4}$ (red), $10^{5}$ (green), $10^{6} M_{\odot}$ (blue). The corresponding black hole mass is shown on the top axis. (b) Average mass of $10^{4} M_{\odot}$ (red) and $10^{6} M_{\text {odot }}$ (blue) impacting clouds captured by the central black hole versus maximum halo circular velocity $(\mathrm{km} / \mathrm{s})$ for a range of braking $(\eta=0.1$, dashed lines $; \eta=0.3$,dot-dash lines, $\eta=1.0$, solid lines). (c) Average fraction of activity in nuclei due to radiatively inefficient accretion versus maximum halo circular velocity $(\mathrm{km} / \mathrm{s})$ for $10^{4} M_{\odot}(\mathrm{red})$ and $10^{6} M_{\odot}$ (blue) impacting clouds for a range of braking $(\eta=0.1-1)$ and a range of cloud densities (see text for details). The horizontal dotted line corresponds to an activity fraction of $1 / 3$ ( $\mathrm{Ho}$ 2008). tering in LINERs Constantin \& Vogeley (2006).

\section{CONCLUSIONS}

In this letter we introduce a new mechanism that delivers large quantities of gas to galactic nuclei on astrophysically interesting timescales. We show that a single warm halo cloud (WHC) impact on a galactic bulge could potentially deliver a large mass $\left(\sim 10^{4-6} M_{\odot}\right)$ of gas to the central regions of a galactic nucleus, in a singular event. Although there are considerable uncertainties in the parameters of warm halo clouds, some representative numbers suggest that the impacts occur on astrophysically interesting timescales and at a rate that must account for some or even all of the low luminosity activity observed in galactic nuclei in the local Universe. Based on analytic $\Lambda \mathrm{CDM}$ calculations of cooling halos, our model predicts an impact rate relatively independent of galaxy mass. Our model also predicts that larger mass black holes $\left(>10^{7} M_{\odot}\right)$ can capture significant fractions of the impacting cloud mass at some critical value depending on the nuclear braking $(\eta)$. At this critical value, our model predicts the highest Eddington ratios. Below this critical mass, our model predicts that most of the cloud mass will not accrete onto the black hole. Instead, this material will mix with the ISM in the nuclear bulge or induce star formation, which may lead to delayed episodes of high Eddington ratio accretion. Finally, our model predicts that, for a reasonable range of cloud masses, densities and braking, the fraction of supermassive black holes accreting at very low Eddington ratios in the local Universe could be around $\sim 1 / 3$, which agrees with observations ( $\mathrm{Ho}$ 2008).

\section{ACKNOWLEDGEMENTS}

BM \& KESF gratefully acknowledge the support of the Department of Astrophysics of the American Museum of Natural History, PSC grant PSC-CUNY-40-397 and CUNY grant CCRI-06-22. AM acknowledges support from an ROA supplement to NSF award AST-0904059. We acknowledge useful discussions with Mordecai-Mark Mac Low and a very helpful report from the anonymous referee.

\section{REFERENCES}

Blitz L., Spergel D.N., Tueben P.J., Hartmann D. \& Burton W.B., 1999, ApJ, 514, 818

Boomsma R., Oosterloo T.A., Fraternali F., van der Hulst J.M. \& Sancisi R., 2008, A\&A, 490, 555

Cavaliere A. \& Vittorini V., 2002, ApJ, 570, 114

Chynoweth K.M., Langston G.I., Yun M.S., Lockman F.J., Rubin K.H.R. \& Scholes S.A., 2008, ApJ, 135, 1983

Ciotti L. \& Ostriker J.P., 1997, ApJ, 487, L105

Ciotti L. \& Ostriker J.P., 2007, ApJ, 665, 1038

Clark P.C., Bonnell I.A., Zinnecker H. \& Bate M.R., 2005, MNRAS, 359, 809

Constantin A. \& Vogeley M.S., 2006, ApJ, 650, 727

Dekel A., Sari R. \& Ceverino D., 2009, ApJ, 703, 785

Doherty M., Bunker A., Sharp R., Dalton G., Parry I. \& Lewis I., 2006, MNRAS, 370, 331

Edmunds M.G., 1990, MNRAS, 246, 678

Fall S.M. \& Efstathiou G., 1980, MNRAS, 193, 189

Ferrarese L., 2002, ApJ, 578, 90

Garcia-Burillo S., Combes F., Schinnerer E., Boone F. \& Hunt L.K., 2005, A\&A, 441, 1011

Gultekin K. et al., 2009, ApJ, 698, 198

Heitsch F. \& Putman M.E., 2009, ApJ, 598, 1485

Ho L., 2008, ARA\&A, 46, 475
Hopkins P.F. \& Hernquist L., 2006, ApJS, 166, 1

Hopkins P.F., Hernquist L., Cox T.J., Robertson B. \& Krause E., 2007, ApJ, 669, 67

Kauffmann G. \& Heckman T.M., 2009, MNRAS, 397, 135

Kawakatu N., Umemura M. \& Mori M., 2003, ApJ, 583, 85

Kerĕs D., Katz N., Weinberg D.H. \& Dave R., 1997, ApJ, 487, 591

Klein R.I., McKee C.F. \& Colella P., 1994, ApJ, 420, 213

Kormendy J. \& Richstone D., 1995, ARA\&A, 33, 581

Krolik J.H., 2001, Active Galactic Nuclei (Princeton University

Press, Princeton, New Jersey)

Kwak K., Shelton R.L. \& Raley E.A., 2009, ApJ, 699, 1775

Lockman F.J., Benjamin R.A., Heroux A.J. \& Langston G.I., 2008, ApJ, 679, 21

Maller A. \& Bullock J. S., 2004, MNRAS, 355, 694

Metzger P.G., Dushcl W.J. \& Zylka R., 1996, A\&AR, 7, 289

Miller E.D., Bregman J.N. \& Waaker B.P., 2009, ApJ, 692, 470

McKernan B., Yaqoob T. \& Reynolds C. S., 2004, ApJ, 617, 232

McKernan B., Yaqoob T. \& Reynolds C. S., 2007, MNRAS, 379, 1359

Nayakshin S., Cuadra J. \& Springel V., 2007, MNRAS, 379, 21

Oosterloo T.A., Morganti R., Sadler E.M., van der Hulst T. \&

Serra P., 2007, A\&A, 465, 787

Ostriker J.P. \& Rees M.J., 1977, MNRAS, 179, 541 
Peek J.E.G., Putman M.E., McKee C.F., Heiles C. \& Stanimirović, 2007, ApJ, 656, 907

Peek J.E.G., Putman M.E. \& Sommer-Larsen, 2008, ApJ, 674, 227

Putman M.E. et al., 2002, AJ, 123, 873

Schartmann M., Burkert A., Krause M., Camenzind M., Meisenheimer K. \& Davies R.I., 2010, MNRAS, astro-ph/0912.4677

Schawinski K., Thomas D., Sarzi M., Maraston C., Kaviraj S., Joo S.-J., Yi S.K. \& Silk J., 2007, MNRAS, 382, 1415

Shapiro P.R. \& Field, G.B., 1976, ApJ, 205, 762

Shankar F., Weinberg D.H. \& Miralda-Escude, J., 2009, ApJ, 690, 20

Shen Y., 2009, ApJ, 704, 89
Strickland D. K., Heckman T.M., Colbert, E.J.M. Hoopes C.G. \& Weaver K.A., 2004, ApJS, 151, 193

Tang S., Wang Q.D., Mac Low M.-M. \& Joung, M. R., 2009, MNRAS, 398, 1468

Thom C., Peek J.E.G., Putman M.E., Heiles C., Peek K.M.G. \& Wilhelm, R., 2008, ApJ, 684, 364

Vittorini V., Shankar F. \& Cavaliere, A., 2005, MNRAS, 363, 1376

Wakker B.P. \& van Woerden, H., 1997, ARA\&A, 35, 217

White S.D.M. \& Frenk C.S., 1991, ApJ, 379, 52

Xu Y.-D., Narayan R., Quataert E., Yuan F. \& Baganoff F. K., 2006, ApJ, 640, 319 